\title{
On the Automation System of Transformer Substation
}

\author{
Liu Zhengdao, Li Xiyuan, Wang Qiqi \\ Yingkou Power Supply Company of National Grid Liaoning Electric Power Co., Ltd \\ jkweiz@sina.com
}

Keywords: The Automation System of Transformer Substation; Structure; Function

\begin{abstract}
This paper offers a brief introduction to the importance and development trend of automation system of transformer substation, proposing the basic concept of integrated automation of transformer substation and analyzing the system structure, communication mode, the basic functions and the development prospect of transformer substation automation.
\end{abstract}

\section{Introduction}

Power grid is an integral whole. The integrated utilization of the primary and secondary device information is of prime importance to the secure and stable operation of power grid. The integrated automation of transformer substation is a measure of increasing the safe, reliable and stable operation level of transformer substation, reducing the operation and maintenance cost, enhancing economic benefits and providing the users with high-quality power services. Along with the rapid development of automation technology, communication technology, computer and network technology, it has become an inevitable trend for the integrated automation system to replace or renew the traditional secondary system of transformer substation; protection also requires more robust functions, such as self-check, fault recording, incident record, operation monitoring and control management. It is also a new trend in the development of electronic power system to develop and improve the integrated automation system of transformer substation.

\section{System Structure}

According to the development situation of the integrated automation of transformer substation, it mainly consists of the following structures

\section{Distributed System Structure}

The multiple computers single-function device distributed according to the monitoring objects of transformer substation and system functions are connected with the network allowing resources sharing for distributed processing. The marked characteristic of system structure is to distribute the functions of automation system of transformer substation to different computers. The distributed model is usually designed according to their functions by means of multi-CPU system working mode, which has enhanced the capacity of handling parallel multiple events and resolved the bottleneck issues of CPU operation processing. Each functional module (usually multiple CPUs) usually adopts the network technology or serial mode to realize data communication and selects the high priority network system to solve the bottleneck issue related to data transmission, thus enhancing the realtime performance of system. The distributed structure facilitates the expansion and maintenance of system with partial failure not influencing the normal operation of other modules. This model can form centralized group panel or layered group panel, which are widely applied to medium and low voltage substation. Since its establishment, the integrated automation system of distributed transformer substation has displayed its great vitality. Currently, it is still plagued by issues, such as anti-electromagnetic interference, information transmission path and reliability guarantee. 


\section{Centralized System Structure}

The centralized system structure usually adopts the computers with strong functions to expand its I/O interface, intensively collect, calculate and process the analog quantity and digital quantity of the transformer substation and separately complete the computer supervisory control, protection and automatic control. Input, output, protection, control and supervision are completed by the front-end processor and data processing, displaying, printing and distant communication undertaken by the background computer. Currently, the transformer substation of our bureau adopts this structure, which has the following deficiencies.

(1) The front-end processor, with multiple leads, has to undertake heavy tasks, thus reducing the reliability of the whole system; the failure of front-end processor will lose the local and remote information and functions.

(2) The complicated software and heavy modification work result in the complicated system debugging.

(3) Due to the inflexible configuration, the software and hardware must be redesigned for the transformer substations with different main wiring or scales; meanwhile, it is also difficult to expand the functions needed by automation.

\section{Layered and Distributed Structure}

The total station control level-substation level (station-level measure and control unit) and the two-level distribution control system of local unit control level-bay level (bay unit) are set according to the control level and object of the transformation substation. It can also be divided into three levels, namely, substation level, communication level and bay level.

Compared with the centralized processing system, this structure is possessed of the following marked advantages

(1) Enhanced Reliability. The failure of any part of the device will simply impact the local device; in other words, it disperses the "danger"; the failure of substation system or network only influences the supervision but ensures the normal operation of the most important protective and control functions at the segment level; the damage of any intelligent unit at the segment level should not result in the communication outage of the total station, for example, the long-time occupation of the communication network of the total station.

(2) The high expandability and openness is conducive to the design and application of project.

(3) The marked reduction of cables needed by the secondary device can save investment and simplify the debugging and maintenance.

\section{Common}

Currently, the Ethernet communication is usually applied in China. However, before the Ethernet communication, both RS-232C and EIA-422/485 cannot avoid the deficiency of complicated communication system and slow communication speed. The application of fieldbus has partially alleviated the demands of the transformer substation automation system for communication; however, it is not that effective when dealing with large system capacity. The application of Ethernet communication has resolved all these communication-related issues. The common communication modes include

1) Double Ethernet communication and double supervisory control computers are mainly applied to the 220-500KV transformer substation; the double control computers+double servers are used to support optical/electrical Ethernet.

2) Single Ethernet, double/single supervisory control computer model.

3) Double LON network, double/single supervisory control computer model. 
4) Single LON network, double/single supervisory control computer model.

\section{The Functions of the Application of Automation System of Transformer Substation \\ Microcomputer Protection:}

It protects all the electrical equipment within the substation, including circuit protection, transformer protection, bus protection, capacitor protection and spare power automatic switching as well as low frequency decrease load. These protections should contain the following functions

1) Failure Logging

2) Storage of Multiple Sets of Constant Values

3) Display and Correct the Constant Value

4) Communicate with the monitoring system. The failure information and action sequence are sent out according to the orders of monitoring system. Value setting and self-diagnosis of signals. Receive the selection of monitoring system or correct the constant value and synchronize the clock. Standard rules should be applied to the communication.

\section{Data Collection and Processing Function}

Include state data, analog data and pulse data.

\section{1) State Quantity Acquisition}

The quantity of state includes: router status, disconnecter status, transformer tap signal and warning signal of primary equipment of transformer substation, resultant signal of fault-trip and anticipating signal. Currently, most of these signals adopt the photoelectric isolation method for system input and can be obtained through communication mode.

\section{2) Analog Acquisition}

The typical analog quantity acquired by the conventional substations includes the bus voltage of each section, line voltage, current and active and reactive power. Feeder current, voltage and active and reactive power.

\section{Accident Record and Failure Recording and Ranging}

The accident record should include the record of protection of action sequence and switch tripping and closing.

The failure recording of the transformer substation could be realized through two means according to the needs; one is the centralized configuration of specialized fault recorder and communication with the monitoring system. Another is the disperse type. The microprocessor protection device could also be used for recording and ranging calculation. Then the digitalized wave mode and ranging structure are sent to monitoring system for storage and analysis.

\section{Control and Operation Functions}

The operators are able to conduct distant operation of the breaker, disconnector, transformer tap and capacitor switching through the background computer screen. To prevent the impossible operation of controlled equipment caused by system failure, the direct artificial tripping and closing should be preserved in system design.

\section{Misoperation-Preventive Locking Function}

\section{Self-diagnosis of the System}

The plug-in in the system shall be possessed of the function of self-diagnosis and able to send the data to the background computer and distant dispatching center. The real-time self-inspection of the equipment ensures the convenient maintenance and repair; at the meantime, the real-time 
inspection, such as query standard input, can be utilized for other parts. It can quickly find the internal failure of the equipment, give indications and point out the failure location.

\section{Data Processing and Recording}

The formation and storage of historical data serve as the main content of data processing, including the data required by the dispatching center of upper level and electric substation management and protection.

1) The action times of breaker;

2) Accumulation of truncation capacity and number of tripping operation at the fault clearing of breaker;

3) The maximum, minimum and time of regular recording of active, reactive and bus voltage of the active, reactive and transformer;

4) The active and reactive load and daily peak-to-valley value and time;

5) Record of the operating control and correction of setting.

According to the needs, this function can be thorough realized in the local transformer substation as well as the remote operating center or dispatching center.

\section{The Self-Diagnosis Function of Man-machine Interface System}

Each plug-in in the system shall be possessed of the self-diagnosis function. The self-diagnosis information, like the data collected, should be regularly sent to the background computer and remote dispatching center or operational control center for the communication with the remote control center.

The remote modification of protection setting and failure recording as well as the teletransmission of ranging signals are added to the regular action "four-remote control system"; whose information amount far exceeds that of the traditional remote-action system. It must also be possessed of the functions of time synchronization with the dispatching center, unified clock and local operational maintenance.

\section{Conclusions}

The aforesaid analysis shows that the integrated automation of power substation has greatly boosted the automation of power grid dispatching and field operation and management and enhanced the security of power grid and economic operation level. With great significance to the safe and stable operation of power grid, it can also strengthen the efficiency and reliability of the primary and secondary system of power grid. Along with the technological progress and improved software and hardware environment, its superiority will inevitably demonstrated in the future.

\section{References}

[1] Liu Yunlong. On the Safe Operation and Management of Integrated Automatic Transformer Substation [J]. Non-State Running Science \& Technology Enterprises, 2010(12).

[2] Fan Zhusheng. The Daily Operation and Maintenance of Integrated Automation System of Transformer Substation [J]. Technology and Market, 2011, (3).

[3] Zhao Feng. On the Safe Operation and Management of Automatic Transformer Substation [J]. Reform and Discussions, 2008 (3).

[4] Yi Qixun. On the Development Trend of Integrated Automation Technology of Transformer Substation.

[5] Wang Haiqiu, He Renmu. Supervising the System Resource Balance of Master Station with the Integrated Automation of Transformer Substation [J]. 